\title{
Molecular cloning and transcriptional regulation of ompT, a ToxR-repressed gene in Vibrio cholerae
}

\author{
Caiyi C. Li, ${ }^{1,2}$ J. Adam Crawford, ${ }^{3}$ Victor J. DiRita ${ }^{3,4}$ \\ and James B. Kaper ${ }^{2,5 *}$ \\ ${ }^{1}$ Department of Biochemistry and Molecular Biology, \\ University of Maryland, Baltimore, School of Medicine, \\ Baltimore, MD 21201, USA. \\ ${ }^{2}$ Center for Vaccine Development, University of \\ Maryland, Baltimore, School of Medicine, Baltimore, \\ MD 21201, USA. \\ ${ }^{3}$ Department of Microbiology and Immunology, University \\ of Michigan Medical School, Ann Arbor, MI 48109, USA. \\ ${ }^{4}$ Unit for Laboratory Animal Medicine, University of \\ Michigan Medical School, Ann Arbor, MI 48109, USA. \\ ${ }^{5}$ Department of Microbiology and Immunology, University \\ of Maryland, Baltimore, School of Medicine, Baltimore, \\ MD 21201, USA.
}

\section{Summary}

In pathogenic Vibrio cholerae, at least 17 genes are co-ordinately regulated by ToxR. Most of these genes, including those that encode cholera toxin (CT), toxin co-regulated pilus (TCP), accessory colonization factor (ACF) and OmpU, are positively regulated. OmpT is the only identified protein under negative regulation of ToxR. To understand the molecular mechanism by which ToxR represses OmpT expression, we cloned ompt and characterized the ompt promoter and its interaction with ToxR. Sequence analysis revealed that ompT encodes a predicted $35.8 \mathrm{kDa}$ outer membrane porin of $\boldsymbol{V}$. cholerae. Primer extension analysis identified a transcriptional start site 104 bp upstream of the translational start codon. Both primer extension analysis and promoter fusion studies showed that ToxR represses OmpT expression at the transcriptional level. Promoter fusion studies also suggest that cyclic AMP receptor protein (CRP) is involved in ompT activation. Gel mobility shift assays combined with DNase I footprinting analysis demonstrated that ToxR mediates repression of ompT transcription by directly binding to an A/T-rich region between -95 and $-\mathbf{3 0}$ of the ompT promoter. To further understand how the interaction of ToxR with different promoters

Received 24 June, 1999; revised 28 September, 1999; accepted 4 October, 1999. *For correspondence at Center for Vaccine Development, University of Maryland, Baltimore, School of Medicine, Baltimore, MD 21201, USA. E-mail jkaper@umaryland.edu; Tel. $(+1)$ 410706 2493; Fax (+1) 4107060182 results in its function as an activator or repressor, we have also mapped the regions on the $\operatorname{ctx} A B$ and toxT promoters to which ToxR binds. The regions protected by ToxR on each of these promoters are all A/T rich and large in size, although they are positioned differently relative to each transcriptional start site.

\section{Introduction}

The Gram-negative bacterium Vibrio cholerae is a common inhabitant of the aquatic environment. Pathogenic strains, when ingested with contaminated water or food, can cause the potentially fatal diarrhoeal disease cholera in humans. Pathogenicity of $V$. cholerae is associated with possession of specific virulence factors and the ability to co-ordinately regulate the expression of these factors in response to environmental stimuli. The best-characterized virulence factors of $V$. cholerae are cholera toxin (CT), a potent exotoxin largely responsible for the characteristic watery diarrhoea (for a review, see Kaper et al., 1995), and the toxin co-regulated pilus (TCP), an essential intestinal colonization factor (Taylor et al., 1987). Recent findings indicated that the genes encoding CT are part of the genome of a lysogenic filamentous phage (CTX $\Phi)$ which uses TCP as a receptor for infection of host strains (Waldor and Mekalanos, 1996). The genes encoding TCP are part of a $40 \mathrm{~kb} \underline{\mathrm{V}}$. cholerae pathogenicity island (VPI) unique to pathogenic V. cholerae strains (Kovach et al., 1996; Karaolis et al., 1998), which was recently shown to be the genome of another phage VPIФ (Karaolis et al., 1999).

ToxR, a transmembrane DNA-binding protein, has been shown to be an important regulator of virulence gene expression in $V$. cholerae. In conjunction with another membrane protein ToxS, ToxR controls expression of at least 17 genes termed the ToxR regulon (Miller and Mekalanos, 1988; Peterson and Mekalanos, 1988). The ToxR regulon is organized into two branches: tox $T$ independent and toxT dependent (Champion et al., 1997). In the former branch, ToxR activates expression of an outer membrane porin and potential adherence factor OmpU (Sperandio et al., 1995, 1996; Chakrabarti et al., 1996), and represses expression of another outer membrane protein OmpT (Miller and Mekalanos, 1988). In the latter branch, ToxR and ToxS act synergistically with another pair of membrane regulators, TcpP and TcpH (Carroll et al., 1997; Häse and Mekalanos, 1998), to activate transcription of tox $T$, which encodes a transcriptional activator belonging 
to the AraC family. ToxT in turn activates expression of $\mathrm{CT}$, TCP and the accessory colonization factor (ACF) (DiRita et al., 1991; Higgins et al., 1992). The toxT gene is encoded on the VPI along with the genes encoding TCP and ACF (Kovach et al., 1996; Karaolis et al., 1998). In Escherichia coli, ToxRS can also directly activate transcription from the $\operatorname{ctx} A B$ promoter (Miller and Mekalanos, 1984), but apparently does not do so in $V$. cholerae (Champion et al., 1997).

ToxR is a $32.5 \mathrm{kDa}$ protein with a single transmembrane domain, an amino-terminal cytoplasmic domain and a carboxy-terminal periplasmic domain (Miller et al., 1987). The cytoplasmic DNA-binding domain of ToxR shares $\approx 30 \%$ homology at the amino acid level with the carboxyterminal DNA-binding domain of $\mathrm{OmpR}$, a porin regulator in E. coli. Therefore, ToxR has been included in the OmpR family of response regulators, members of which bind to DNA by a winged helix-turn-helix motif (Pratt and Silhavy, 1996; Martínez-Hackert and Stock, 1997). ToxS has been hypothesized to interact with ToxR in the periplasm and stabilize an active state of ToxR (DiRita and Mekalanos, 1991; Pfau and Taylor, 1998). The lack of a phosphoacceptor domain in ToxR distinguishes ToxR from OmpR and other two-component response regulators. In addition, OmpR is a cytoplasmic transcription factor and ToxR is a transmembrane DNA-binding protein. Recently, more regulatory proteins sharing the topological and functional features of ToxR have been described, including TcpP of $V$. cholerae. Other such regulators include CadC of $E$. coli, which regulates lysine decarboxylase expression (Neely et al., 1994), PsaE of Y. pseudotuberculosis, which regulates fimbrial expression (Yang and Isberg, 1997), and ToxR homologues of Vibrio fischeri, Vibrio parahaemolyticus and Photobacterium sp. strain SS9 (Lin et al., 1993; Reich and Schoolnik, 1994; Welch and Bartlett, 1998). Each of these proteins has a ToxS-like protein associated with their activity except for CadC. Thus, ToxR has been recognized as the prototype of a unique class of membrane-associated regulators whose mechanism of action is poorly understood.

Previous studies that examined the DNA-binding properties leading to transcription activation by ToxR have focused on the $c t x A B$, toxT and ompU promoters. The distinguishing feature of the $\operatorname{ct} A B$ promoter is a heptad, TTTTGAT, located at -56 relative to the transcription start site and directly repeated 3-8 times depending on the V. cholerae strain (Mekalanos et al., 1983). Deletion studies on the $c t x A B$ promoter revealed that at least three copies of the heptad are required for ToxR binding and activation of transcription (Miller et al., 1987). Studies using an in vivo footprinting approach recently showed that the TTTTGAT motif is not sufficient for ToxR-mediated activation because ToxR did not bind to a synthetic target having only three direct repeats without flanking sequences (Pfau and Taylor, 1996). The toxT promoter, however, is characterized by three inverted repeats spanning from -172 to -49 relative to the transcription start site; disruption of one of these inverted repeats $(-93$ to -58$)$ abolished transcription activation by ToxR (Higgins and DiRita, 1994). In a PCR mutagenesis study, two point mutations that abolish ToxR binding were mapped to the upstream region of inverted repeat 3 . However, when symmetrical nucleotides in the downstream half site were mutated, no effect on ToxR binding or activation was observed, indicating that ToxR does not recognize this DNA sequence as an inverted repeat per se (Higgins and DiRita, 1996). ToxR also directly interacts with the ompU promoter at three sites: an upstream site ranging from -238 to -139 , and two downstream sites ranging from -116 to -58 and from -53 to -24 (Crawford et al., 1998). The common feature of the sequences interacting with ToxR on these promoters is their $A / T$ richness, although no consensus ToxR-binding site could be identified from these studies.

OmpT is the only recognized member of the ToxR reguIon that is repressed by ToxR, yet little is known about OmpT regulation and function during pathogenesis. Here, we describe the molecular cloning of ompT and studies on the mechanism of ompT repression by ToxR. We also present DNase I footprinting data that identifies the ToxRbinding site on the promoters of the ompT, ctxAB and tox $T$ promoters. How ToxR functions both as an activator and as a repressor in the context of different promoters is discussed.

\section{Results}

Cloning and expression of the ompT gene

OmpT was previously recognized as a $40 \mathrm{kDa}$ outer membrane protein whose expression is greatly increased in a toxR mutant (Fig. 1, lanes 2 and 5) (Miller and Mekalanos, 1988). In order to clone the ompT gene, we separated the outer membrane proteins of a tox $R$ mutant of $V$. cholerae 395, denoted CVD639, by SDS-PAGE. The proteins were then transferred to a PVDF membrane from which the band corresponding to OmpT was excised and used to obtain the $\mathrm{N}$-terminal amino acid sequence. A search using the BLAST computer program revealed that the $\mathrm{N}$ terminal 20 amino acids of OmpT share strong homology with the $\mathrm{N}$-terminal sequences of outer membrane porins OmpF, OmpC and PhoE of E. coli (Fig. 2). This result is consistent with the previous observations that $\mathrm{OmpU}$ and OmpT are porin-like proteins of $V$. cholerae (Chakrabarti et al., 1996).

Based on $V$. cholerae codon usage and the highly conserved regions of the $E$. coli porin genes, a degenerate oligonucleotide was designed from the OmpT sequence (Fig. 2). Strong homology with $E$. coli K-12 sequences prevented

(c) 2000 Blackwell Science Ltd, Molecular Microbiology, 35, 189-203 


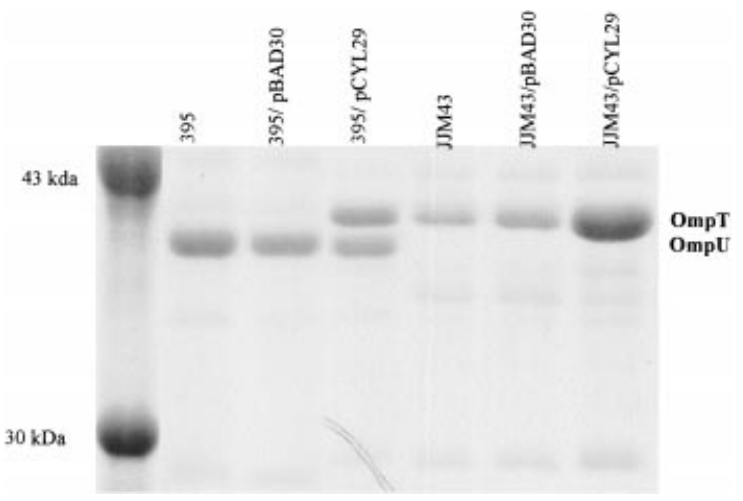

Fig. 1. Overexpression of OmpT in $V$. cholerae. Plasmid pCYL29 containing ompT under the control of the $\mathrm{P}_{\mathrm{BAD}}$ promoter and the parental vector pBAD30 were introduced into $V$. cholerae 395 and its tox $R^{-}$mutant JJM43. Outer membrane proteins were prepared after induction for $2 \mathrm{~h}$ by $0.2 \%$ arabinose at mid-log phase, separated on a $14 \%$ SDS polyacrylamide gel and visualized by staining with Coomassie brilliant blue. The positions of OmpT and OmpU are indicated.

the use of this oligonucleotide as a probe in directly screening a $V$. cholerae library in $E$. coli. Instead, we used it as a primer in an anchored PCR procedure to amplify a $2.2 \mathrm{~kb}$ fragment from the $V$. cholerae 395 chromosome. The amplified fragment was then used as a probe to screen a cosmid library of $V$. cholerae 395, ultimately leading to the cloning and sequencing of the complete ompT gene as described in Experimental procedures.

None of our cosmid clones contained an intact ompT gene, suggesting that expression of ompT under its own promoter might be toxic in E. coli. To verify this possibility, we cloned the structural gene of ompT under the control of the $P_{B A D}$ promoter to obtain plasmid pCYL29. pCYL29 was introduced into $E$. coli $\mathrm{DH} 5 \alpha$ and $V$. cholerae strains. Upon induction with $0.2 \%$ arabinose, $\mathrm{DH} 5 \alpha$ (pCYL29) was rapidly lysed, whereas both the wild type and the tox $R$ mutant of $V$. cholerae could tolerate OmpT overexpression (Fig. 1). The failure of our attempts so far in constructing an ompT deletion mutant strain of 395 suggests that OmpT is essential for the survival of this $V$. cholerae strain. The presence of omp $T$ in all $V$. cholerae strains examined, both pathogenic and non-pathogenic (data not shown), is also consistent with ompT being an essential gene in this species.

\section{Sequence analysis of ompT}

Analysis of the complete ompT sequence revealed several features of the predicted OmpT protein sequence and potential regulatory elements. Figure 3 shows the $\mathrm{N}$-terminal coding region and the regulatory region of the ompT sequence. The ompT open reading frame consists of $975 \mathrm{bp}$ and encodes a predicted protein of 325 amino acids $(35.8 \mathrm{kDa})$, although it appears as a $40 \mathrm{kDa}$ band on an SDS-PAGE gel. A typical signal peptide of 19 amino acids precedes the mature protein and the predicted first 20 amino acid residues of the mature protein match exactly the experimentally determined $\mathrm{N}$-terminal sequence. The promoter elements were predicted according to the consensus sequence of $E$. coli $\sigma^{70}$ promoters and verified by primer extension analysis (see below). The presence of a sequence likely to be a rho-independent terminator after the stop codon suggests ompT is monocistronically transcribed.

Comparison of the predicted OmpT sequence with the SWISSPROT data base revealed that many outer membrane proteins of enteric bacteria share significant homology with OmpT. Despite the homology between OmpT and E. coli porins at the $\mathrm{N}$-terminus, the complete ompT sequence is most closely related (with $53 \%$ similarity and $30 \%$ identity at protein level, $60 \%$ identity at nucleotide level) to $\mathrm{ompH}$ of deep-sea Photobacterium sp. strain SS9 (Bartlett and Welch, 1995).

\section{Primer extension analysis of ompT transcription}

Primer extension analysis was carried out to map the $5^{\prime}$ terminus of the ompT transcript and to measure the relative amount of ompT mRNA in wild-type strain 395 and its tox $R$ mutant CVD639. Cells were grown in LB medium at $37^{\circ} \mathrm{C}$ to mid-log phase. Total RNA was extracted and used in primer extension reactions as described in the Experimental procedures. As shown in Fig. 4, a single primer extension product for ompT is observed in both strains, suggesting a single transcription start site, which is located $104 \mathrm{bp}$ upstream of the translational start codon. Such a long untranslated leader sequence, which might be involved in stabilizing mRNA, was also observed for ompU in V. cholerae (Sperandio et al., 1996) and the

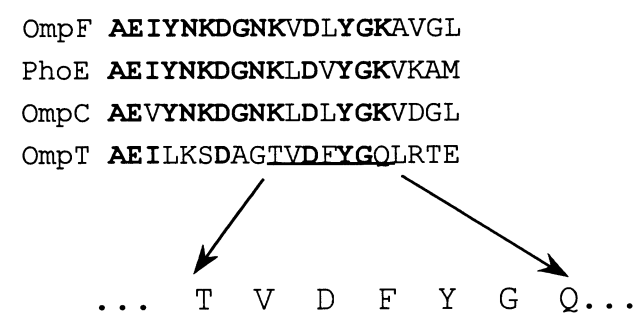

Degenerate primer $5^{\prime}$ GGCACCGTCGATTTCTACGG $3^{\prime}$

$$
\begin{array}{llll}
\mathrm{T} & \mathrm{T} & \mathrm{T} & \mathrm{T} \\
& \mathrm{A} & \\
& \mathrm{G} &
\end{array}
$$

Fig. 2. Sequence alignments of the $\mathrm{N}$-terminal regions of OmpT and three E. coli porins $\mathrm{OmpF}, \mathrm{OmpC}$ and PhoE. Bold letters indicate the residues that are conserved in at least three sequences. The residues used in designing the degenerate oligonucleotide are underlined and shown along with the sequence of the degenerate primer. 
-530 ACAAAGGGTTTTGGCCATCTCGGCAACTAGGAAATTTTGAGCGCGACGAG GAATGAAATTTTCCAATTGATTTTGTAGATTTTGATAACTGTTGCGAATG GAATCTTGAATGTTTTTTGTCAGCATAGAGGTGTCCAAAGTACGATGGCG CGATGGTAGCÁCAGATCACTGAGTGATACCTACTTCACGAÄATCGTATGC

-330 TTTTAATAGAAATCTAGATCTTGTTCACAAATAAAAACTGAACCTTCAGA GACTTAAATTTATTTTGTTTTATAAAAAATÁTATAGCTCCAAATCCTAGG TTTGATTTTTAंTTAATATCCTAATTTCGCCAंTTTAAATGTTAATTTTTGG ÄGTTTTATTCTGGTTTTCTGTCGGGTTGTAATCCTGTGATTTCGTCTGTT

-130 TTTGTTAAGAACTAAGCCGAAAAAAATCATGAAATAAATGTAATTTATTG • $\cdot \begin{array}{r}\cdot \\ \text { CRP site } 2 \\ -35\end{array}$

AATTTTAAGGTTTTATGTTTTTTTCTTTGTTTTTTTTATGGTATTTGACA \begin{tabular}{cccc}
\hline & $-10 \quad \cdot \quad+1$ & $\cdot$ \\
TGCAGAATATTCTTTTGCAATCTTAACCACGAATCTAATGGCGGTGGTGA & CRP site 3
\end{tabular} CCAAACAAAGAGTTGGAAAACCACCTTCAAAAAACAGGGAACCGGACAAG +70

GAATTCCCATATTAAGAAAAGGCAGTGGATTAACATGAAAAAAACTCTAT $\begin{array}{lllll}M & K & K & T & L\end{array}$

TAGCACTCGCAGTGCTTGCAGCCGCAGGCTCTGTAAACGCAGCTGAAATT

$\begin{array}{lllllllllllllllll}L & A & L & A & V & L & A & A & A & G & S & V & N & A & A & E & I\end{array}$ CTAAAATCAGATGCTGGCACTGTTGATTTTTACGGTCAGCTACGTACAGA

$\begin{array}{lllllllllllllllll}\mathrm{L} & \mathrm{K} & \mathrm{S} & \mathrm{D} & \mathrm{A} & \mathrm{G} & \mathrm{T} & \mathrm{V} & \mathrm{D} & \mathrm{F} & \mathrm{Y} & \mathrm{G} & \mathrm{Q} & \mathrm{L} & \mathrm{R} & \mathrm{T} & \mathrm{E}\end{array}$
Fig. 3. Partial sequence of $o m p T$. The transcriptional start site, -10 and -35 regions of the ompT promoter and a predicted ribosome-binding site (RBS) are in bold. Underlined are three putative CRP binding sites centred at $-310,-85$ and -7 . The coding region of a signal peptide (italic) and the first 20 amino acids are shown. The complete sequence of ompT was deposited in GenBank with accession number AF 079766. porin genes in E. coli (Emory and Belasco, 1990). Promoter elements of $o m p T$ were then deduced according to the transcriptional start site. The -35 element perfectly matches the $\sigma^{70}$ prokaryotic consensus sequence (TTGACA), but the -10 region (TGCAAT) varies from the consensus (TATAAT) in two base pairs. In addition to the poor -10 region, the spacing between the -35 and -10 region is $15 \mathrm{bp}$, which is less than the optimal $17 \mathrm{bp}$ spacing, indicating that $o m p T$ may not have a strong core promoter.

In addition to providing a transcriptional start site, the result of the primer extension experiment indicates that ToxR represses ompT expression at the transcriptional level because a much higher level of ompT primer extension product is observed in CVD639 compared with 395 (Fig. 4).

\section{Activation and repression of transcription from ompT promoter fusions}

To localize the cis-acting elements required for the activation and repression of $o m p T$ transcription, various portions of the ompT promoter region were cloned in front of a promoterless lac $Z$ gene on the broad host range plasmid pTL61T (Linn and Pierre, 1990), and $\beta$-galactosidase activities were measured for each fusion construct. The $3^{\prime}$ end of all the constructs was chosen at +104 , immediately in front of the translational start. The $5^{\prime}$ end points of the fusion constructs are: $-490,-323,-165$ and -44 relative to the transcriptional start.

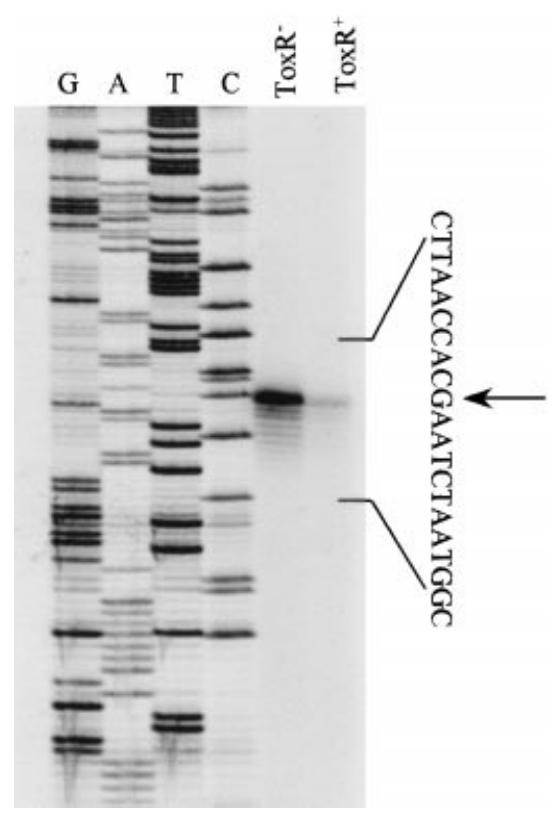

Fig. 4. Primer extension analysis of $o m p T$ mRNA. RNA was isolated from $V$. cholerae wild-type $395\left(\right.$ ToxR $\left.^{+}\right)$or tox $R^{-}$mutant CVD639 (ToxR ${ }^{-}$) during mid-log phase of cell growth at $37^{\circ} \mathrm{C}$. A radiolabelled primer was incubated with $30 \mu \mathrm{g}$ of RNA for each strain and reverse transcriptase was added to produce a DNA copy of the transcripts, which were analysed on a $6 \%$ sequencing gel. The same primer was used to prime dideoxy-sequencing products with a DNA template containing the ompT promoter region. The sequence adjacent to the transcription start site $(G$, indicated by an arrow) is shown to the side of the figure.

(c) 2000 Blackwell Science Ltd, Molecular Microbiology, 35, 189-203 
(A) JJM43

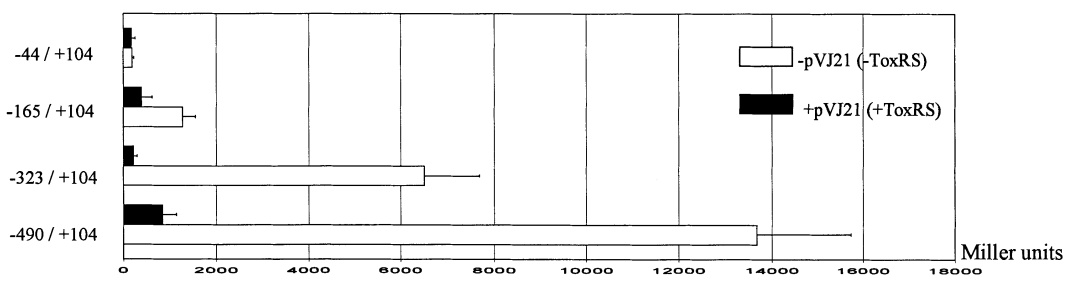

(B) 395

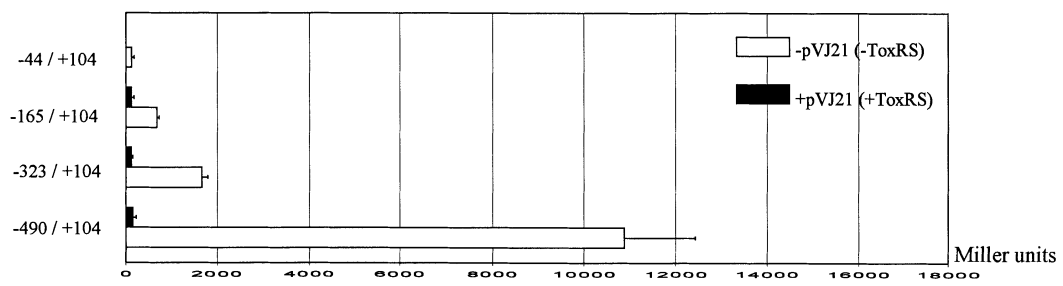

Fig. 5. $\beta$-Galactosidase activities (Miller units) of ompT-lacZ fusions in tox $R^{-}$mutant JJM43 (A) and wild-type 395 (B). The $5^{\prime}$ and $3^{\prime}$ endpoints of the ompT promoter in each fusion are labelled relative to the ompT transcription start site. Open bars indicate ompT promoter activity in the absence of a ToxRS-expressing plasmid, whereas solid bars indicate ompT promoter activity in the presence of a plasmid encoding ToxRS. Data in each chart represent the average of at least three independent measurements.
To measure transcription activity from the ompT promoter in the absence of ToxR, the fusion constructs were introduced into the tox $R$ mutant $V$. cholerae strain JJM43, in which a high level of OmpT expression was previously observed (Miller and Mekalanos, 1988). As shown in Fig. 5A (compare all the open bars only), the smallest fusion, -44 to +104 , directs very low levels of $\beta$-galactosidase activity, consistent with the prediction based on sequence analysis that ompT does not have a strong core promoter. Extension of the $5^{\prime}$ end of the fusion to -165 brings approximately sixfold activation of transcription, and further extension to -490 brings another 10fold activation. The requirement of so much upstream DNA for high-level transcription of ompT in the absence of ToxR suggests that one or more additional factors is involved in ompT activation.

To demonstrate that ToxR can repress transcription from these plasmid-borne ompT promoter fusions, ToxR and ToxS were then provided by a plasmid encoding wildtype ToxRS (pVJ21). Despite different levels of activation, transcription was dramatically repressed for all the constructs except the minimal construct -44 to +104 (Fig. $5 \mathrm{~A}$, compare open and filled bars for each construct), indicating that some sequence elements downstream of -165 can mediate ToxR-dependent repression.

The fusion constructs were also introduced into wildtype strain 395 to quantify the repression of transcription from the plasmid-borne ompT promoter by endogenous levels of ToxR. Differences in $\beta$-galactosidase activities of the plasmid-borne fusions between JJM43 and 395 (for each fusion, compare open bars in Fig. 5A with those in $B)$ are not as dramatic as the differences in the OmpT protein and mRNA levels between these two strains (see Figs 1 and 4). To account for this, we hypothesized that multiple copies of the ompT operator site were titrating endogenous levels of ToxR because the plasmid used to construct these fusions is a pBR322 derivative with a copy number of 15-20. We tested this hypothesis by introducing pVJ21 into 395 carrying the various deletion constructs. When ToxR was overexpressed this way, complete repression was observed (Fig. 5B, filled bars), consistent with the hypothesis that the presence of multicopy ompT promoter saturates the endogenous level of ToxR, resulting in insufficient repression.

As cyclic AMP receptor protein (CRP) binding sites were predicted within the ompT promoter region based on sequence comparison (Fig. 3) and a previous study (Skorupski and Taylor, 1997) has shown that CRP is involved in the regulation of the virulence genes in the ToxR regulon, we tested the hypothesis that CRP is involved in the regulation of ompT. As shown in Fig. 6, when transcription from different promoter fusions was monitored in a crp mutant strain of 395 , denoted KSK377, the full-length construct $-490 /+104$ lost most of its activity, which was recovered when complemented with a plasmid containing crp (pCYL47). The $-165 /+104$ construct, which is activated sixfold compared with the core promoter, was not activated (perhaps even slightly repressed) by CRP. These results suggest two levels of activation for the ompT promoter: the first level involving a cis-acting element between -44 and -165 and the second level involving CRP, which presumably binds to the CRP binding site centred at -310 . The large region of DNA involved in the activation of this promoter suggests the regulation of the omp $T$ transcription is complex and the exact role of CRP in this regulation deserves further investigation.

\section{ToxR binds specifically to DNA upstream of ompT}

To determine whether ToxR represses the otherwise very 


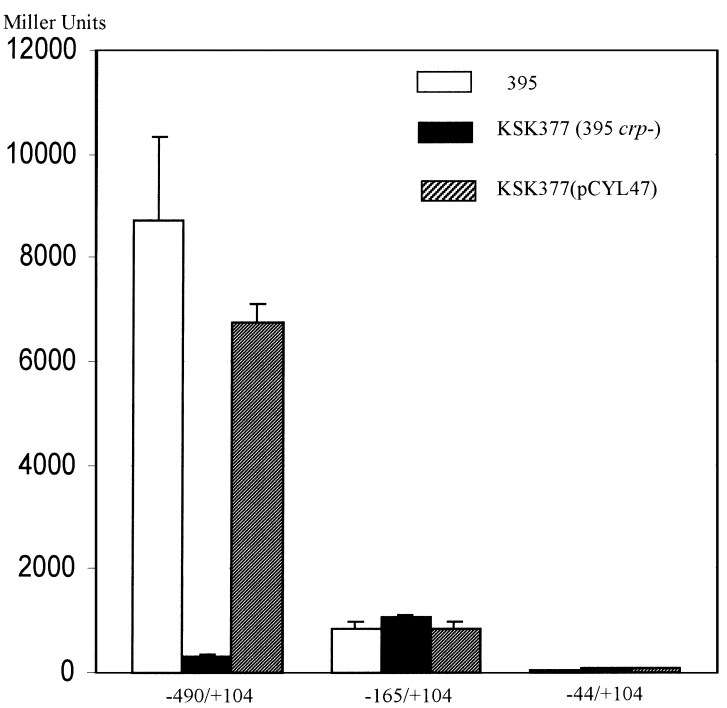

Fig. 6. CRP is involved in the activation of ompT. $\beta$-Galactosidase activity from different $o m p T-l a c Z$ fusions was measured in wildtype V. cholerae 395 (open bars), crp mutant KSK377(solid bars) and KSK377 containing pCYL47 encoding CRP (hatched bars). Data in this chart represent the average of at least three independent measurements. strong ompT transcription through direct interaction with the ompT promoter, gel electrophoretic mobility shift assays were performed on different fragments of the ompT promoter as previously described (Higgins and DiRita, 1994). DNA fragments extending from -323 to $+104,-165$ to +104 and -44 to +170 relative to the ompT transcription start site were radiolabelled and incubated with varying amounts of membrane fractions from $E$. coli carrying the ToxRS $^{+}$plasmid pVJ21 or the cloning vector pACYC184 as ToxRS $^{-}$control. As shown in Fig. 7A, the -323 to +104 fragment was shifted by ToxRS-containing membranes, but the -44 to +170 fragment was not, indicating that ToxR does not bind to the region downstream of the core promoter. The $5^{\prime}$ end of the ToxR-binding site was further narrowed down to -165 because the amount of membrane that completely shifts the -323 to +104 fragment can also completely shift the -165 to +104 fragment (Fig. 7B), indicating that the sequence element downstream of -165 is sufficient to mediate Tox $R$ binding. These results are consistent with the lacZ fusion experiments described above, showing that transcription repression by ToxR can be mediated by sequences downstream of -165 .
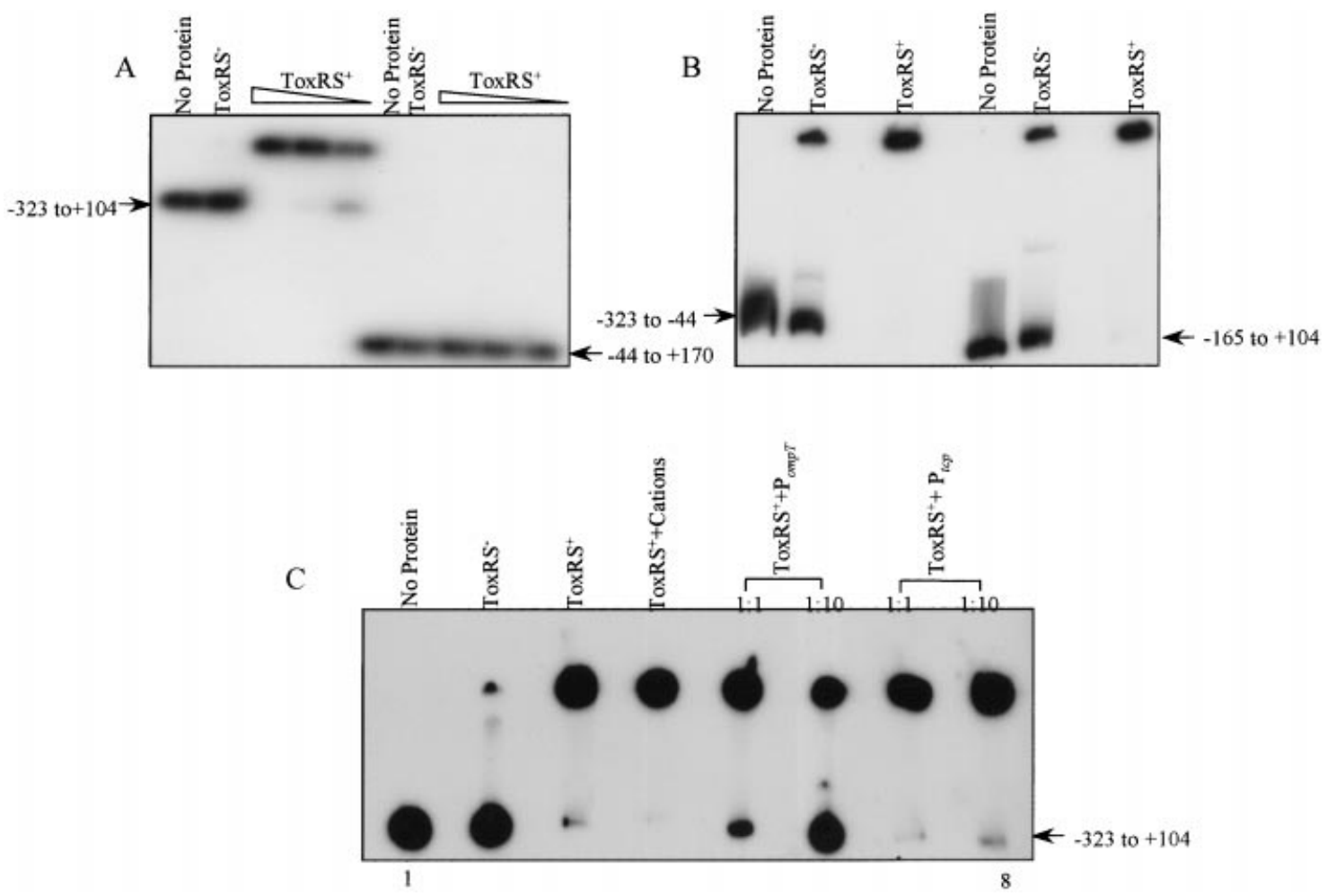

Fig. 7. Specific binding of ToxR to the ompT promoter. DNA fragments containing different regions of the ompT promoter $(-323$ to +104 and -44 to +170 in $A ;-323$ to +104 and -165 to +104 in $B$ ) were radiolabelled and used as a probe in the gel mobility shift assays. The probes were incubated with ToxRS-containing membranes $\left(\right.$ ToxRS $^{+}$) or negative control membranes (ToxRS ${ }^{-}$). In C, the -323 to +104 probe was used. + pomp $T$ and + ptcpA indicate competition assays in which unlabelled ompT $(-323$ to +104$)$ or tcpA promoter fragments were added such that the molar ratio of probe to cold competitor was $1: 1$ or $1: 10$. +Cations indicates that $\mathrm{Ca}^{2+}$ and $\mathrm{Mg}^{2+}$, which are required in the DNase I footprinting assay, were included in the binding reaction. The presence of these cations does not affect ToxR binding. Note that because all the shifted complexes are located at the wells of the gel, quantification was carried out by comparing the amount of unshifted probes. 
We noticed that when a larger amount of probe is used in the gel shift assay, a higher background binding to the membranes without ToxR was observed (compare Fig. $7 \mathrm{~A}$, where 3000 c.p.m. probe was used, with $\mathrm{B}$, where 20000 c.p.m. was used), although only membranes containing ToxR can completely shift the probes. To demonstrate the specificity of the interaction between ToxR and the ompT promoter, a competition assay using unlabelled ompT and tcpA promoter sequences was performed (Fig. 7C). The tcpA promoter is not regulated by ToxR directly and ToxR does not cause an electrophoretic mobility shift of labelled tcpA promoter DNA (Champion et al., 1997; Ron Taylor, personal communication). When unlabelled -323 to +104 fragment of the ompT promoter DNA was added to the binding reaction with the same fragment labelled as probe, an increase in the amount of unshifted probe was observed. In contrast, when unlabelled tcpA promoter sequence was added to a similar binding reaction, no competition was observed. We conclude that ToxR binding to the ompT promoter is specific.

\section{ToxR footprint on the ompT promoter}

To further elucidate the mechanism of transcription repression mediated by ToxR binding to the ompT promoter, we performed DNase I footprinting analysis. A restriction fragment corresponding to the -165 to +104 region of the ompT promoter, which harbours the putative ToxR-binding site as suggested by the transcriptional fusion studies as well as the gel mobility shift experiments, was radiolabelled on one strand and incubated with $E$. coli membranes containing ToxRS. The binding reactions were next treated with DNase I as described previously (Crawford et al., 1998) and subjected to electrophoresis on a denaturing polyacrylamide gel to identify the region protected from DNase I digestion as a result of ToxR binding. Although the DNase I cleavage pattern generated in the presence of negative control membranes $\left(\right.$ ToxRS $^{-}$) is identical to that of DNA alone (data not shown), membranes containing ToxRS $\left(\right.$ ToxRS $^{+}$) protected a large region corresponding to the -30 to -95 region of the ompT promoter (Fig. 8A). The large size of the ToxR foot-
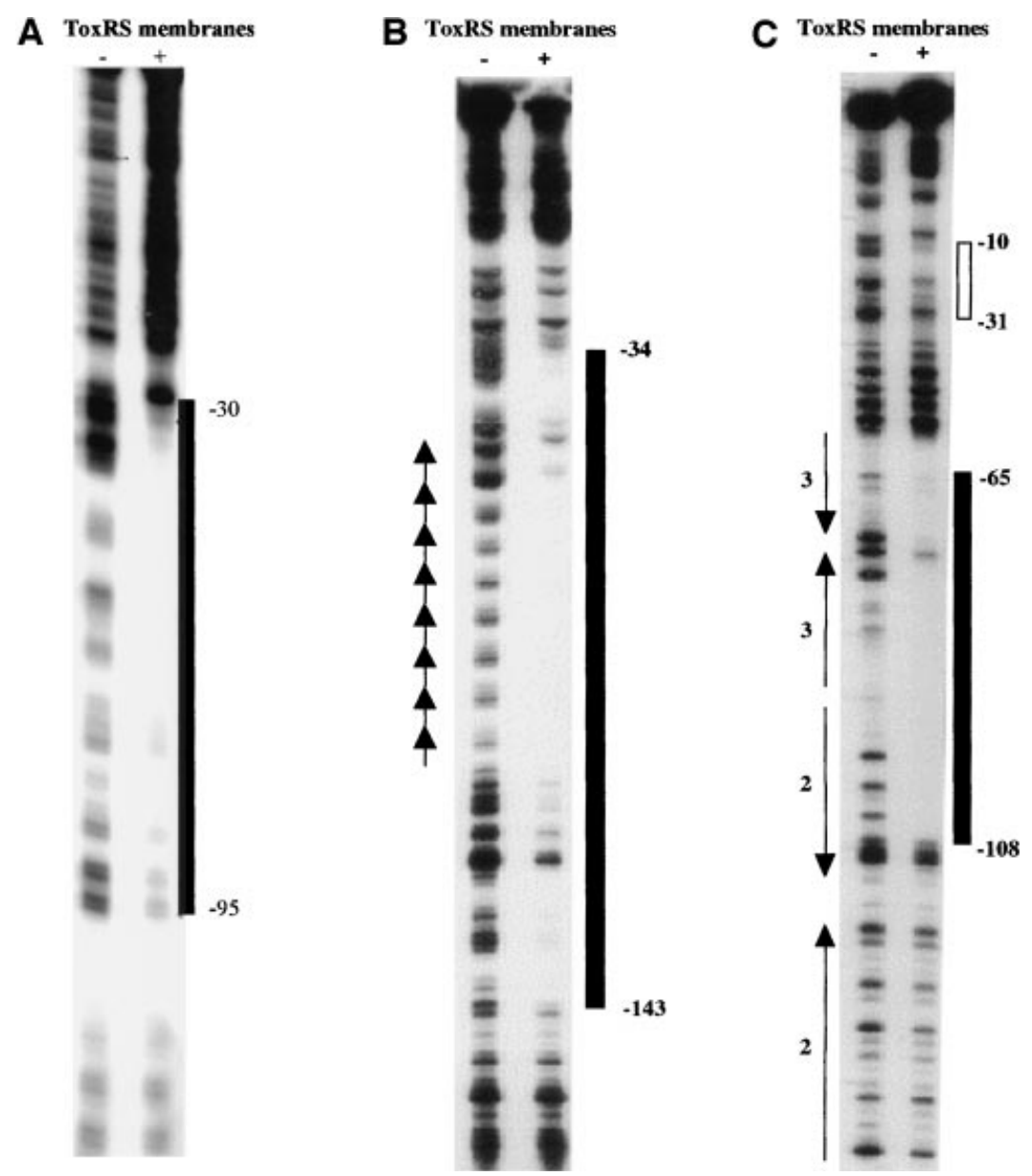

Fig. 8. DNase I footprints of ToxR on the $\operatorname{ompT}(\mathrm{A}), \operatorname{ctx} A B(\mathrm{~B})$ and toxT $(\mathrm{C})$ promoters. Radiolabelled restriction fragments of each promoter were subjected to DNase I footprinting as described in the Experimental procedures. Labels at the top indicate whether $E$. coli membranes without ToxRS $(-)$ or with ToxRS (+) were used. Reactions in $A$ and $C$ contained $1000 \mu \mathrm{g} \mathrm{ml}^{-1}$ and those in B contained $2000 \mathrm{\mu g} \mathrm{ml}^{-1}$. The bars to the side represent the regions on each probe protected by ToxR and are labelled relative to the transcription start site. The open bar next to the tox $T$ footprint indicates weak protection. Arrows to the side of $B$ and $C$ represent the direct repeats present in the $\operatorname{ct} x A B$ promoter and the inverted repeats present in the toxT promoter. 
print $(60 \mathrm{bp})$ suggests that multiple ToxR molecules are involved in binding. Although this region does not seem to contain the motif previously identified in the $\operatorname{ctx} A B$ and to $T$ promoters, the sequence itself is characterized by strings of Ts interrupted by invariant Gs. In fact, the whole ToxR footprint in ompT can be represented as five tandem repeats of $T G(a / T)_{3} T T T N N$, which can also be identified in the binding sites for ToxR in the $\operatorname{ctx} A B$ and tox $T$ promoters (see below), but the presence of this sequence element is not obvious in the ToxR-binding site within the ompU promoter (Crawford et al., 1998). Whether this $10 \mathrm{bp}$ element is what is recognized by ToxR in the context of the ompT promoter remains to be further examined.

\section{ToxR footprints on the ctxAB and toxT promoter}

As previously discussed, ompT is the only gene known to be repressed by ToxR, whereas at least three other genes, $c t x A B$, toxT and $o m p U$, are directly activated by ToxR. Of the three promoters that ToxR directly binds, the footprint has been determined only for the ompU promoter (Crawford et al., 1998). To help clarify how the binding of ToxR to different promoters leads to activation of some yet repression of others, we performed footprinting studies on the $\operatorname{ctx} A B$ and toxT promoters to identify the ToxRbinding sites within them.

Restriction fragments corresponding to -181 to +7 of the $c t x A B$ promoter region (containing eight copies of the direct repeat) or to -172 to +45 of the tox $T$ promoter region were used in the DNase I footprinting procedure as described above. ToxR protected a relatively large region in the $c t x A B$ promoter from -143 to -34 (Fig. 8B). The eight TTTTGAT repeats are included in this protected region, as are two additional $A / T$-rich regions that flank both sides of these repeats. This is consistent with previous genetic evidence that flanking sequences in addition to the TTTTGAT repeats are required for ToxR binding (Pfau and Taylor, 1996). Analysis of the toxT promoter (Fig. 8C) revealed that ToxR protects a region from -108 to -65 , covering a majority of the A/T-rich region of what has been termed inverted repeat 3 (Higgins and DiRita, 1994) and a portion of the downstream half of inverted repeat 2. Previous data have demonstrated that inverted repeat 3 is critical for ToxR-mediated activation of the toxT promoter (Higgins and DiRita, 1996). Additionally, ToxR weakly protects an A/T-rich region extending from -31 to -10 , the significance of which is unclear.

Based on the data from this study and previous observations on the ompU promoter (Crawford et al., 1998), the exact position of each ToxR footprint on four of its known target promoters as well as the actual sequences are summarized in Fig. 9. Because of the large size and the $A / T$-richness of these sequences, there are multiple ways to align the footprinting sequences of the different promoters, rendering it difficult to derive a consensus sequence. Further dissection of the whole footprints into fragments smaller yet sufficient for ToxR binding may facilitate the identification of the consensus sequence for ToxR recognition.

\section{Discussion}

We report here the molecular cloning of the gene encoding a $V$. cholerae outer membrane porin OmpT, the only member of the ToxR regulon that is negatively regulated by ToxR. We obtained evidence leading to a model in which ToxR represses high-level ompT transcription by binding directly to a region just upstream of the ompT promoter. Although activation of this promoter remains to be fully elucidated, our preliminary results indicate that CRP is involved. Our results on ToxR footprints of the ompT, $C t x A B$ and toxT promoters, together with the previously reported ToxR footprint data on the ompU promoter, provide $a$ basis for further understanding of how ToxR functions differently at different promoters. An interesting question raised by this study is how ToxR manages to recognize seemingly different $A / T$-rich sequences on each of its four target promoters.

\section{ToxR as a regulator of the porin regulon of V. cholerae}

Cloning of the ompT gene confirms the emerging recognition that ToxR, OmpU and OmpT are components of the porin regulon of $V$. cholerae (Champion et al., 1997). Through regulating the relative amount of different porin proteins in response to the environmental signals, a bacterium balances the osmotic strength of the cytoplasm with that of the external milieu, maintains membrane integrity and regulates its ability to obtain nutrients in the medium. This kind of regulation may be essential to pathogenic $V$. cholerae, which in its life cycle must adjust quickly to the changes from the aquatic environment to the intestinal environment and vice versa. Given that ToxR functions as a regulator for porins and is present in non-pathogenic as well as pathogenic strains of $V$. cholerae, ToxR probably has evolved to control outer membrane expression in an ancestral $V$. cholerae strain. When virulence genes on the VPI and CTX $\Phi$ were acquired through horizontal transfer, ToxR may have achieved the ability to regulate these genes by acquiring control of ToxT. By exploiting ToxR (and other regulatory systems of the host) for the regulation of newly acquired virulence genes, a new $V$. cholerae strain may have greater survival advantage because it can efficiently correlate new gene expression with environmental cues. Although extremely low levels of OmpT are expressed in classical strain 395 under laboratory conditions (Fig. 1) and OmpT is thought to be regulated in

(c) 2000 Blackwell Science Ltd, Molecular Microbiology, 35, 189-203 
A
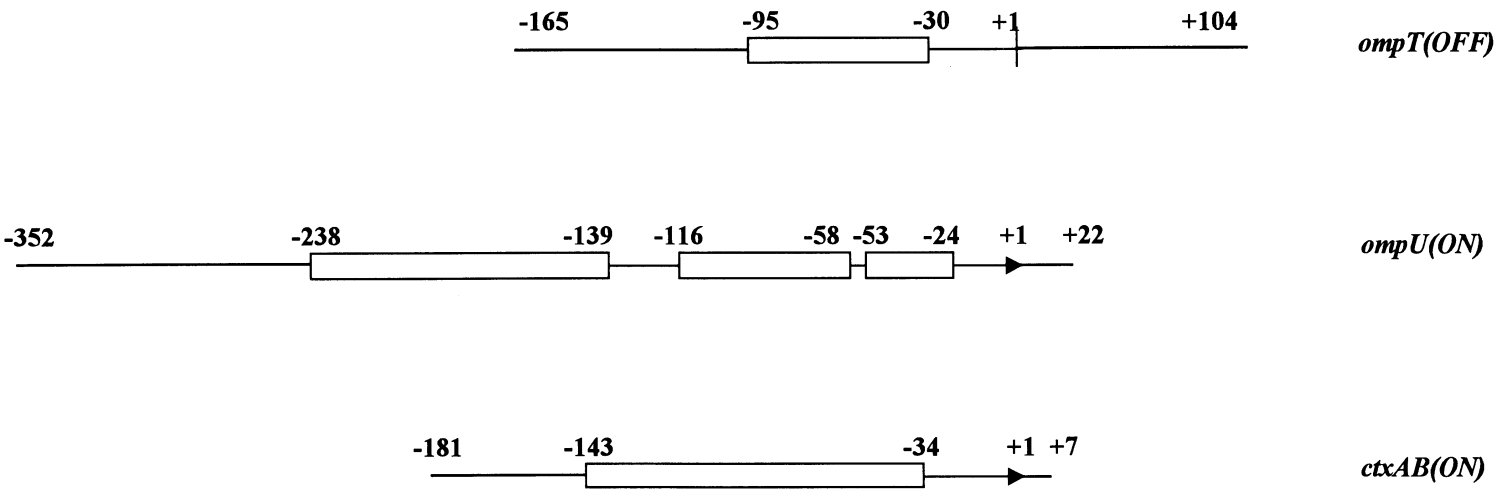

$\operatorname{ctx} A B(O N)$

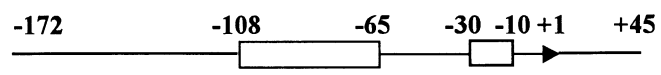

$\operatorname{toxT}(\mathrm{ON})$

B

ompT

$\begin{array}{ll}-95 & -30\end{array}$

AAATGTAATTTAT TGAATTTTAAGGTTTATGTTTTTTCTTTGTTTTTTTTATGGTATTTGACAT

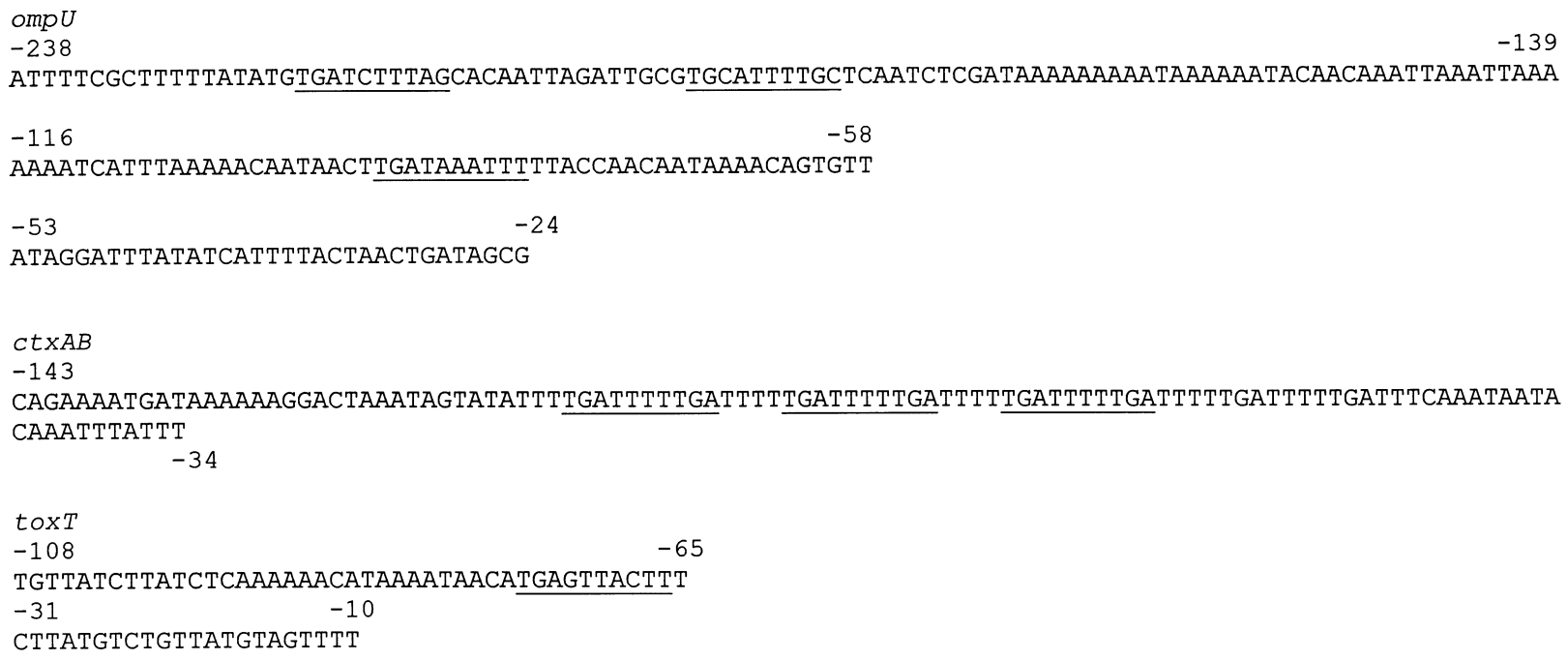

Fig. 9. Summary of the ToxR footprint on each target promoter: ompT, ompU, ctxAB and toxT. A. Schematic representation of the DNA fragments used in the footprinting assay for each promoter. The fragments chosen were the smallest fragments tested that were sufficient to mediate in vitro gel shift and in vivo activation or repression. The rectangles within these fragments represent regions protected by ToxR from DNase I in the footprinting studies; numbers indicate the position relative to the transcription start site. Also indicated are the states (ON or OFF) of each promoter in vivo. B. Nucleotide sequences within the ToxR-protected region (shown as rectangles in A). The five $10 \mathrm{bp}$ repeats TG(a/T) ${ }_{3}$ TTTNN on the ompT and other promoters are underlined.

a fashion opposite to how CTX and TCP are regulated (Miller and Mekalanos, 1988), post-challenge sera from volunteers infected with this strain strongly recognize OmpT as well as OmpU (Sperandio et al., 1996), suggesting that OmpT level may be upregulated in vivo.

The classic porin regulon is the $\mathrm{OmpR} / \mathrm{OmpF} / \mathrm{OmpC}$ system of $E$. coli, which is also a paradigm for the two- component regulatory systems (Slauch and Silhavy, 1996), yet there are crucial differences between OmpR regulation of OmpF/OmpC and ToxR regulation of OmpU/OmpT. These include differences in subcellular localization and the lack of evidence of phosphorylation as a signal relay mechanism controlling ToxR function. In addition, OmpR is required for activation of both $\mathrm{OmpF}$ 
and $\mathrm{OmpC}$ as well as repression of OmpF, depending on the state of phosphorylation, whereas ToxR is only required for the activation of $\mathrm{OmpU}$ and the repression of OmpT. In a tox $R$ mutant strain, OmpT is expressed at a very high level. Interestingly, the porin regulation pattern in $V$. cholerae is very similar to the ToxR/OmpL/OmpH system in Photobacterium sp. strain SS9. OmpL and $\mathrm{OmpH}$ are outer membrane proteins regulated in an opposite fashion by a protein homologous to ToxR ( $50 \%$ identity and $64 \%$ similarity) in response to hydrostatic pressure (Welch and Bartlett, 1998). In a Photobacterium toxR mutant, only $\mathrm{OmpH}$ is expressed. At the amino acid level, OmpT is most closely related to $\mathrm{OmpH}(30 \%$ identity and $53 \%$ similarity) whereas OmpU shares 50\% identity and $63 \%$ similarity to OmpL (Sperandio et al., 1996; Welch and Bartlett, 1998). Thus, the mechanism by which ToxR and its homologues regulate porin gene expression appears quite different from how OmpR functions in E. coli. Interestingly, the OmpR homologue of $V$. cholerae has recently been cloned from strain 569B (Tow and Coyne, 1999). Based on the high degree of similarity shared by the OmpR sequences in $E$. coli and $V$. cholerae, it is possible that OmpR is involved in the porin regulation in $V$. cholerae in addition to ToxR, although no data on this have been reported.

\section{Transcription activation and repression of ompT}

This study provides important insights into the regulation of the ompT promoter. Our data demonstrate that the core promoter for ompT is not active and that a high level of transcription in the absence of ToxR requires a large region of the upstream DNA. Specifically, a fragment harbouring the core promoter, the -10 and -35 elements, with a $5^{\prime}$ end-point at -44 was insufficient to direct $\beta$-galactosidase expression. Upstream sequences up to -490 from the transcriptional start were required for full activation of the promoter. Considering the large size of the promoter region involved, it is likely that another factor(s) is involved in the activation of the ompT promoter.

Our preliminary data suggest that there are two levels of activation of this promoter. The first level is mediated by a cis-acting element between -44 and -165 . Because this whole region, starting immediately upstream of -44 , is very $A / T$ rich, it is possible that this sequence directly interacts with RNA polymerase and serves as an UP element in activating transcription (Ross et al., 1993). There is also the possibility of a trans-acting factor binding to this region. Regulatory mechanisms have been proposed for other pathogenesis systems in which it is predicted that temperature or other environmental parameters encountered on entering the host may alter the topology of the DNA and involve the action of proteins such as the nucleoid-associated protein $\mathrm{H}-\mathrm{NS}$, which binds $\mathrm{A} / \mathrm{T}$-rich
DNA (Atlung and Ingmer, 1997). Generally, H-NS is associated with transcription silencing, not activation, but a recent report details $\mathrm{H}$-NS-mediated activation of the maltose regulon (Johansson et al., 1998). Another regulatory protein that binds $A / T$-rich sequences is integration host factor (IHF), which can induce a sharp turn at the binding site and cause structural perturbation that favours transcription activation at the downstream region or bring the activators or upstream activation sequence elements closer to the RNA polymerase through DNA looping (Bai and Somerville, 1998; Sheridan et al., 1998).

The second level of activation of this promoter is mediated by the sequence between -165 and -490 , in which CRP is apparently involved. A CRP binding site was identified within this region, centred at -310 . We noticed that in the absence of the upstream sequence CRP slightly represses transcription of the -165 to +104 fusion (Fig. 6), and two putative CRP binding sites can be found within this region centred at -85 and -7 respectively. Clearly, CRP has a complicated role in the regulation of this promoter. Since in wild-type $V$. cholerae 395, OmpT is predominantly repressed, it is tempting to speculate that CRP function under certain conditions to allow a higher level of expression of OmpT. Further studies are required to fully elucidate the nature of CRP involvement in the regulation of this promoter.

No matter what the activation mechanism, ToxR evidently interferes with activation of the ompT promoter through binding to a site corresponding to the -30 to -95 region of the promoter. At this position, ToxR can compete for the binding of the activator(s), interfere with the interaction between the activator and RNA polymerase, or interfere with RNA polymerase binding to the basal elements.

\section{Mechanism of differential regulation of ToxR on different promoters}

ToxR functions both as a transcription activator of $c t x A B$, toxT and $o m p U$ and as a repressor of $O m p T$ by direct binding to these promoters. One common feature of all the promoters is the large size of the ToxR footprint (Fig. 9), indicating multiple ToxR molecules are involved in binding to the four target promoters. The individual sequence context and the relative positioning of the ToxR binding sites in each promoter may determine the nature of interaction between ToxR and the transcriptional machinery, ultimately leading to differential regulation of each promoter.

For the ompT promoter, as discussed above, by binding to a region overlapping the -35 element of the promoter, ToxR tightly represses this otherwise highly active promoter. Whether this is achieved simply by competing for the activator or RNA polymerase binding site or by proteinprotein interaction with the activator or the RNA polymerase is not known. For the activation of $o m p U$, according 
to the model proposed by Crawford et al. (1998), a stronger ToxR binding site predicted to reside upstream of -128 is first bound by ToxR, followed by co-operative binding to weaker downstream binding sites down to -24 . Binding at this position is then predicted to facilitate direct interaction with RNA polymerase to activate transcription (Crawford et al., 1998). We notice that on the ompU and ompT promoter the $3^{\prime}$ end of the ToxR footprints are positioned similarly relative to the transcriptional start site (both overlapping the -35 region). Therefore, there are at least two possible explanations for the opposite effects of ToxR on these two promoters. One possibility is that ToxR bound to the ompT promoter interacts with a different region of the RNA polymerase from that when bound to the ompU promoter, or different surfaces of the ToxR are involved in interaction with RNA polymerase. A second possibility is that the sequence differences between the ompU and ompT promoters potentiate ToxR differently, so that it functions to either stabilize or destabilize the intermediates of RNAP-DNA complex during transcription.

In the case of $\operatorname{ctx} A B$, ToxR binding sites are positioned upstream of -35 and activation is postulated to be through direct contact with RNA polymerase, based on analysis of mutations in the $r p O A$ gene encoding the alpha subunit of RNAP (S. R. Cendrowski and V. J. DiRita, unpublished). ToxR activation of $c t x A B$ is only observed in $E$. coli from plasmid-expressed ToxR, and not in $V$. cholerae from endogenous expression (Champion et al., 1997). One explanation for this observation is that the affinity of ToxR to the binding sites on the $c t x A B$ may be sufficiently weak so that overexpression of ToxR is required to achieve binding and activation. The copy number effect observed in the repression of ompT by ToxR (see Fig. 5) is consistent with this explanation. Another possible explanation for the observation that ToxR appears to activate $c t x A B$ only in $E$. coli is that other factors in $E$. coli interact with ToxR for the activation of the $\operatorname{ct} A B$ promoter.

For the activation of toxT, the ToxR binding site encompasses the -108 to -65 region of the promoter, where ToxR may interact with another transmembrane transcription activator TcpP. TcpP binds to a region downstream of the ToxR binding site $(-58$ to -44$)$, where it is hypothesized to interact directly with RNA polymerase (E. S. Krukonis and V. J. DiRita, manuscript in preparation).

These observations suggest that, in addition to the interaction with DNA, ToxR is capable of interactions with other transcription factors as well as differential interactions with RNA polymerase.

\section{The nature of ToxR-DNA interaction}

Although the footprints of ToxR on all four of the ToxR target promoters are now available, the large size and the $A / T$ richness of these sequences make it difficult to derive a single strong consensus ToxR binding site. Consistent with the sequence homology between the DNAbinding domains of ToxR and OmpR family activators, a similar lack of consensus binding site has been observed in promoters regulated by this class of activator in other systems (Drapal and Sawers, 1995; Pratt and Silhavy, 1996). For this family of transcription factors, multiple copies of the protein are usually involved in co-operative binding (Maeda and Mizuno, 1990; Shen and Gunsalus, 1997). DNase I footprinting studies performed in the present study can provide information on the regions that are maximally occupied by multiple ToxR on each promoter, but not on the minimal sequence element that is recognized by a single ToxR molecule. This limitation contributes to the inability to derive a consensus sequence. On the other hand, the difficulty in identifying a consensus sequence for the OmpR/ToxR family of regulators might be revealing the unique character of the activities under their control. A single optimal binding site of a regulator usually mediates a rapid on/off binding, which leads to an all-or-none switch of gene expression. The lack of a single optimal binding site implies that such a sharp on/off switch mechanism is not applicable in these systems, in which a gradual reversible response to a constantly changing parameter such as osmolarity is desirable. Accordingly, identification of a minimal consensus sequence for high-affinity ToxR binding by in vitro selection might identify a sequence not likely to be present in any of its natural promoters. In addition, the use of suboptimal binding sites for one regulator on a promoter provide the possibility of modulating the expression of this gene by multiple factors through protein-protein interaction, which make it possible for the bacteria to fine tune its gene expression in response to multiple environment cues.

The possibility that the DNA-binding domain of ToxR (and other OmpR-like regulators) recognizes a structural element of the DNA in addition to, or perhaps instead of, a specific primary sequence may also account for the fact that ToxR-regulated promoters are not closely related at the nucleotide sequence level. Evidence for this comes from the DNase I digestion pattern of the ToxR-binding regions in the $c t x A B$ and toxT promoters. DNase I clearly recognizes these regions less efficiently relative to the rest of the promoter fragment (see negative control reactions, Fig. $8 \mathrm{~B}$ and $\mathrm{C}$ ), suggesting that they are less accessible to the enzyme. This is perhaps due to distortions in the minor groove where DNase I recognizes and binds DNA (Suck, 1994). That ToxR binds and protects these regions is consistent with the hypothesis that it may recognize distorted DNA.

In conclusion, the present study provides important insight into the molecular mechanism of ToxR-mediated 
Table 1. Vibrio cholerae strains and plasmids used in this study.

\begin{tabular}{|c|c|c|}
\hline Strains or plasmids & Description & Source or reference \\
\hline \multicolumn{3}{|l|}{ Strains } \\
\hline \multicolumn{3}{|l|}{ V. cholerae } \\
\hline 395 & O1 classical & Laboratory collection \\
\hline CVD639 & 395, toxR::bla, $\mathrm{Ap}^{\mathrm{R}}$ & L. E. Comstock and J. B. Kaper (unpublished) \\
\hline JJM43 & 395, $\Delta c t x A B \Delta$ toxR43, $\mathrm{Sm}^{\mathrm{R}}$ & Herrington et al. (1988) \\
\hline KSK377 & 395, crp::kan & Skorupski and Taylor (1997) \\
\hline \multicolumn{3}{|l|}{ Plasmids } \\
\hline pACYC184 & Low copy number cloning vector, Tet $^{R} \mathrm{Cm}^{R}$ & New England Biolabs \\
\hline pBAD30 & Expression vector with inducible pBAD promoter & Guzman et al. (1995) \\
\hline pBluescript SK $+1-$ & High copy number cloning vector, $A p^{R}$ & Stratagene \\
\hline pCYL28 & Promoter region of ompT cloned into pUC18 & This study \\
\hline pCYL29 & Promoterless omp $T$ cloned into pBAD30 & This study \\
\hline pCYL47 & pACYC184; tet::crp & This study \\
\hline pGEM-T & PCR cloning vector, $\mathrm{Ap}^{\mathrm{R}}$ & Promega \\
\hline pTL61T & Promoterless lac $Z$ transcriptional fusion vector; $A p^{R}$ & Linn and Pierre (1990) \\
\hline pUC18 & High copy number cloning vector, $A p^{R}$ & Gibco BRL \\
\hline pVJ21 & pACYC184; tet::toxRS & Higgins and DiRita (1994) \\
\hline pLS716 & pBluescript SK +I-; pctxAB; $\mathrm{Ap}^{\mathrm{R}}$ & This study \\
\hline pTLI2 & pTL61T; ptoxT::lacZ; $\mathrm{Ap}^{\mathrm{R}}$ & Higgins and DiRita (1994) \\
\hline
\end{tabular}

transcription activities and deepens our understanding of the diverse functions of ToxR as not only an important regulator for virulence gene expression in $V$. cholerae but also the prototype of a unique class of transmembrane DNA-binding transcription factor.

\section{Experimental procedures}

\section{Strains and plasmids}

The E. coli and $V$. cholerae strains and plasmids used in this study are listed in Table 1. Strains were maintained at $-70^{\circ} \mathrm{C}$ in Luria-Bertani (LB) medium containing $30 \%$ glycerol. All strains were grown at $37^{\circ} \mathrm{C}$ in LB medium, unless otherwise specified. Antibiotics were used at the following concentrations: ampicillin, $100 \mu \mathrm{g} \mathrm{ml}^{-1}$ (except for the selection of pBAD and its derivatives at $30 \mu \mathrm{g} \mathrm{ml}^{-1}$ ); chloramphenicol, $30 \mu \mathrm{g} \mathrm{ml}^{-1}$ for E. coli, $10 \mu \mathrm{g} \mathrm{ml}^{-1}$ for $V$. cholerae. Plasmids were introduced into all strains by electroporation.

\section{DNA manipulations}

Plasmid DNA was purified with Qiagen columns (Qiagen). PCR was performed using Taq Polymerase (Gibco BRL) as specified by the manufacturer. PCR products were purified by agarose gel electrophoresis followed by gel extraction with the QIAEX II gel extraction system (Qiagen). Cloning was performed using standard protocols as described previously (Sambrook et al., 1989). Double-stranded sequencing of plasmid DNA was performed at the Biopolymer Laboratory of the University of Maryland, Baltimore.

\section{OmpT purification and $\mathrm{N}$-terminal sequencing}

After overnight growth in LB medium at $37^{\circ} \mathrm{C}, V$. cholerae cells were harvested and passed through a French press.
The outer membrane fraction was isolated by differential centrifugation (Chakrabarti et al., 1996). Outer membrane proteins were separated on a $14 \%$ SDS-PAGE gel and blotted onto PVDF membrane $(0.2 \mu \mathrm{m}$, Bio-Rad). OmpT was identified as a $40 \mathrm{kDa}$ protein whose expression was increased in the toxR mutant CVD639. Excised membrane containing OmpT was sent for N-terminal microsequencing at the Macromolecular Structure Facility, Michigan State University.

\section{Cloning strategies}

A degenerate primer K604 (5'-GGYACYGTNGATTTYTACGG-3') was designed from amino acids 9-15 (GTVDFYG) of OmpT and used as one primer in anchored PCR procedures. Specifically, $2.5 \mu \mathrm{g}$ of $V$. cholerae 395 chromosomal DNA was digested with Dral, EcoRV or Pvull, purified and ligated into $10 \mu \mathrm{g}$ EcoRV digests of pBluescript II SK in $20 \mu$ l reactions, which were then diluted 10-fold. One microlitre of each of the diluted reactions was used as the PCR template in a $100 \mu \mathrm{l}$ reaction containing $200 \mathrm{ng} \mathrm{K604}$ and $50 \mathrm{ng}$ of either the T3 or the T7 primer from pBluescript. The amplifications were performed using 2.5 units of Taq polymerase (Gibco $\mathrm{BRL})$. The cycle parameters were as follows: denaturation at $94^{\circ} \mathrm{C}$ for $30 \mathrm{~s}$, annealing at $50^{\circ} \mathrm{C}$ for $45 \mathrm{~s}$, elongation at $72^{\circ} \mathrm{C}$ for $4 \mathrm{~min}$; except for an initial denaturation of $5 \mathrm{~min}$ and a final extension time of $10 \mathrm{~min}, 30$ cycles were used. K604 and T7 amplified a $2.2 \mathrm{~kb}$ fragment from the Pvull digest. This fragment was purified from an agarose gel and radiolabelled to screen a $V$. cholerae 395 gene bank by colony hybridization. One positive cosmid was obtained, which does not contain the complete ompT gene but from which the coding region of ompT, starting from the amino acid 9 of the mature protein, was subcloned as a Hpal/Sspl fragment into pUC18, resulting in pCYL9. Based on the sequence of pCYL9, another oligonucleotide K645 (5'-CTTGACTTGAGAATCGTG-3') was designed to prime toward the upstream regulatory region of ompT and used in another anchored PCR and screening procedure to obtain a second cosmid clone, 
from which the regulatory region of $o m p T$ was subcloned into pUC18 to generate pCYL28. The sequence of the complete ompT gene was derived from pCYL9 and PCYL28 and has been submitted to the GenBank data base under accession number AF079766. To clone the complete ompT gene in pBAD30, the same Hpal/Sspl fragment of pCYL9 was first cloned into the Smal site of pBAD30 to obtain pCYL11, followed by insertion of an EcoRI fragment containing the ribosomal binding site but not the promoter of ompT from pCYL28.

The crp gene under the control of its own promoter was amplified from $V$. cholerae 395 with primers K1669 5'-GGATCCATACCGTGATCATGTGCAC-3' and K1670 5' -CCCGGGTCTTCGACCATGGCTGATA-3' and cloned into the tet gene of pACYC184 to obtain PCYL47.

\section{Primer extension}

RNA was isolated from a mid-log phase $V$. cholerae culture using Trizol Reagent (Gibco BRL). Ten picomoles of primer (5'-GCACTGCGAGTGCTAATAGA-3') was end-labelled using $50 \mu \mathrm{Ci}$ of $\left[\gamma^{-32} \mathrm{P}\right]-A T P$ and T4 kinase (Gibco BRL) as described previously (Sambrook et al., 1989). Approximately $2 \mathrm{pmol}$ of labelled primer was added to $30 \mu \mathrm{g}$ of RNA, and DEPC (diethylpyrocarbonate) water was added to a final volume of $20 \mu \mathrm{l}$. The mixtures were incubated at $70^{\circ} \mathrm{C}$ for $10 \mathrm{~min}$ and then at $42^{\circ} \mathrm{C}$ to allow annealing of the primer to the template. The extension reaction was then carried out with the SuperScript Preamplification System for First Strand cDNA Synthesis (Gibco BRL), as specified by the manufacturer. Extended reaction mixtures were resolved in $8 \%$ denaturing polyacrylamide gels and visualized by autoradiography following standard protocols (Sambrook et al., 1989).

\section{Transcription fusion analysis}

PCR products harbouring various portions of the ompT promoter were generated with flanking $X b a l$ and HindlII sites and cloned into the high copy number PCR cloning vector pGEM-T (Promega). The cloned fragments were confirmed to have no point mutation by DNA sequencing. These fragments were digested with $X b a l$ and HindIII and cloned into pTL61T. The fusion plasmids were electroporated into $V$. cholerae 395, 395 (pVJ21), JJM43, JJM43 (pVJ21), KSK377 or KSK377 (pCYL47). Strains harbouring fusion plasmids were grown overnight in LB medium containing appropriate antibiotics at $37^{\circ} \mathrm{C}$, then diluted $1: 100$ in fresh medium and grown for $2-3 \mathrm{~h}$ at $37^{\circ} \mathrm{C}$. The cells were then harvested and $\beta$-galactosidase activity was measured as previously described (Miller, 1972).

\section{DNA gel mobility shift assay}

E. coli membrane fractions containing ToxR and ToxS were prepared as previously described (Higgins and DiRita, 1994; Miller et al., 1987). OmpT promoter fragments subcloned into pGEM-T were isolated as DNA probes by first digesting plasmid DNA with Notl, followed by end-labelling the linearized plasmids in a reaction containing dATP, dGTP or dTTP each at $2 \mathrm{mM},\left[\alpha^{-}{ }^{32} \mathrm{P}\right]$-dCTP $\left(>3000 \mathrm{Cimmol}^{-1}\right)$ and 5 units of Klenow (Gibco BRL). Reactions were incubated at room tem- perature for $5 \mathrm{~min}$ followed by $70^{\circ} \mathrm{C}$ for $15 \mathrm{~min}$. The samples were then digested with $\mathrm{Ncol}$, which cuts the probe fragment away from the vector as single end-labelled probe. The probe was then electrophoresed on a $5 \%$ polyacrylamide gel and subjected to autoradiography. Probe fragments were excised out of the gel and eluted at $37^{\circ} \mathrm{C}$ in $0.5 \mathrm{M}$ ammonium acetate, $\mathrm{pH} 7.5,0.1 \%$ SDS, $1 \mathrm{mM}$ EDTA, pH 8.0, followed by ethanol precipitation. Retardation of radiolabelled DNA fragments was performed as previously described (Ottemann et al., 1992), except that the binding reactions were incubated at $30^{\circ} \mathrm{C}$.

\section{DNase I footprinting}

The omp T footprinting probe, which contains -165 to +104 of the promoter region, was synthesized and isolated as described in the DNA gel mobility shift procedure. The $c t x A B$ footprinting probe, which contains -181 to +7 of the promoter region, was generated by cutting pLS716 with Sall followed by an end-labelling reaction containing dATP, dGTP or dTTP, each at $2 \mathrm{mM}, 30 \mu \mathrm{Ci}$ of $\left[\alpha-{ }^{32} \mathrm{P}\right]-\mathrm{dCTP}\left(>3000 \mathrm{Cimmol}^{-1}\right.$, Amersham) and 5 units of Klenow (New England Biolabs) for $5 \mathrm{~min}$ at room temperature followed by $70^{\circ} \mathrm{C}$ for $15 \mathrm{~min}$. The probe fragment was cut out of pLS716 with Sacl. The tox $T$ footprinting probe, which contains -172 to +45 of the promoter, was generated by cutting PTLI2 with Sall followed by an end-labelling reaction as described for the $c t x A B$ footprinting probe. The probe fragment was cut out of pTLI2 using BamHI. Probe fragments were isolated as described above in the DNA gel mobility shift procedure. DNase I footprinting reactions were performed in $70 \mu$ l volumes containing $10 \mathrm{mM}$ Tris-HCl, pH 7.4, 1 mM EDTA, pH 8.0, $50 \mu \mathrm{g} \mathrm{ml}^{-1}$ BSA, $5 \mathrm{mM} \mathrm{NaCl}, 50 \mathrm{mM} \mathrm{KCl}, 0.01 \mathrm{mg} \mathrm{ml}^{-1}$ salmon sperm DNA, 70000 c.p.m. probe, and either 1000 or $2000 \mu \mathrm{g} \mathrm{ml}^{-1}$ of either negative control membranes (ToxRS ${ }^{-}$membranes) or experimental membranes (ToxRS ${ }^{+}$membranes). Reactions were incubated at $30^{\circ} \mathrm{C}$ for $30 \mathrm{~min}$. Under these conditions, $100 \%$ of the probe is bound by ToxR, as measured by electrophoretic mobility shift assays. After the binding reactions, $\mathrm{CaCl}_{2}$ and $\mathrm{MgCl}_{2}$ were added to final concentrations of $1 \mathrm{mM}$ and $5 \mathrm{mM}$ respectively. DNase I (0.01 units; Boehringer Mannheim) was next added and incubated at room temperature for $2 \mathrm{~min}$. The reactions were stopped by addition of a stop solution consisting of $200 \mathrm{mM} \mathrm{NaCl}, 2 \mathrm{mM}$ EDTA and $1 \%$ SDS, followed by three phenol-chloroform extractions. Reactions were then ethanol precipitated, washed twice with $70 \%$ ethanol and electrophoresed on a $5 \%$ polyacrylamide $6.9 \mathrm{M}$ urea gel. To map precisely the position of the ToxR binding region in each promoter, a sequencing reaction was performed (Amersham Life Sciences, T7 Sequenase Version 2.0 DNA sequencing kit) on the appropriate strand of pLS716, pTLI2 or pCYL28 in which the $5^{\prime}$ end of the sequencing primer corresponds to the $5^{\prime}$ end of the footprinting probe. The sequencing reaction was electrophoresed alongside the footprinting reactions.

\section{Nucleotide sequence analysis and GenBank accession number}

Sequence analysis was performed using the GCG (Genetics Computer Group) software package. The complete sequence of ompT has been assigned GenBank accession number AF 079766. 


\section{Acknowledgements}

We thank R. Taylor for providing KSK377 and S. Adhya for insightful discussions. This work was supported in part by grants Al 19716 (to J.B.K.), Al 31645 (to V.J.D.), Al 45125 (to V.J.D.) and RR-00200 (to the Unit for Laboratory Animal Medicine, University of Michigan) from the National Institute of Health. J.A.C. is a Rackham Predoctoral Fellow of the University of Michigan and a trainee of the University of Michigan Genetics Training grant 5T32 GM 07544.

\section{References}

Atlung, T., and Ingmer, H. (1997) H-NS: a modulator of environmentally regulated gene expression. Mol Microbiol 24: 7-17.

Bai, Q., and Somerville, R.L. (1998) Integration host factor and cyclic AMP receptor protein are required for TyrRmediated activation of $t p /$ in Citrobacter freundii. J Bacteriol 180: 6173-6186.

Bartlett, D.H., and Welch, T.J. (1995) ompH gene expression is regulated by multiple environmental cues in addition to high pressure in the deep-sea bacterium Photobacterium species strain SS9. J Bacteriol 177: 1008-1016.

Carroll, P.A., Tashima, K.T., Rogers, M.B., DiRita, V.J., and Calderwood, S.B. (1997) Phase variation in tcpH modulates expression of the ToxR regulon in Vibrio cholerae. Mol Microbiol 25: 1099-1111.

Chakrabarti, S.R., Chaudhuri, K., Sen, K., and Das, J. (1996) Porins of Vibrio cholerae: purification and characterization of OmpU. J Bacteriol 170: 2575-2583.

Champion, G.A., Neely, M.N., Brennan, M.A., and DiRita, V.J. (1997) A branch in the ToxR regulatory cascade of Vibrio cholerae revealed by characterization of tox $T$ mutant strains. Mol Microbiol 23: 323-331.

Crawford, J.A., Kaper, J.B., and DiRita, V.J. (1998) Analysis of ToxR-dependent transcription activation of $o m p U$, the gene encoding a major envelope protein in Vibrio cholerae. Mol Microbiol 29: 235-246.

Drapal, N., and Sawers, G. (1995) Purification of ArcA and analysis of its specific interaction with the pfl promoterregulatory region. Mol Microbiol 16: 597-607.

DiRita, V.J., and Mekalanos, J.J. (1991) Periplasmic interaction between two membrane regulatory proteins, ToxR and ToxS, results in signal transduction and transcriptional activation. Cell 64: 29-37.

DiRita, V.J., Parsot, C., Jander, G., and Mekalanos, J.J. (1991) Regulatory cascade controls virulence in Vibrio cholerae. Proc Natl Acad Sci USA 88: 5403-5407.

Emory, S.A., and Belasco, J.G. (1990) The ompA 5' untranslated RNA segment functions in Escherichia coli as a growth-rate-regulated mRNA stabilizer whose activity is unrelated to translational efficiency. $J$ Bacteriol 172: 44724481.

Guzman, L.M., Belin, D., Carson, M.J., and Beckwith, J. (1995) Tight regulation, modulation, and high-level expression by vectors containing the arabinose $P_{B A D}$ promoter. $J$ Bacteriol 177: 4121-4130.

Häse, C.C., and Mekalanos, J.J. (1998) TcpP protein is a positive regulator of virulence gene expression in Vibrio cholerae. Proc Natl Acad Sci USA 95: 730-734.
Herrington, D.A., Hall, R.H., Losonsky, G., Mekalanos, J.J., Taylor, R.K., and Levine, M.M. (1988) Toxin, toxin-coregulated pili, and the ToxR regulon are essential for Vibrio cholerae pathogenesis in humans. J Exp Med 168: 14871492.

Higgins, D.E., and DiRita, V.J. (1994) Transcriptional control of toxT, a regulatory gene in the ToxR regulon of Vibrio cholerae. Mol Microbiol 14: 17-29.

Higgins, D.E., and DiRita, V.J. (1996) Genetic analysis of the interaction between Vibrio cholerae transcription activator ToxR and toxT promoter DNA. J Bacteriol 178: 1080-1087.

Higgins, D.E., Nazareno, E., and DiRita, V.J. (1992) The virulence gene activator ToxT from Vibrio cholerae is a member of the AraC family of transcriptional activators. $J$ Bacteriol 174: 6974-6980.

Johansson, J., Dagberg, B., Richet, E., and Uhlin, B.E. (1998) H-NS and StpA proteins stimulate expression of the maltose regulon in Escherichia coli. $J$ Bacteriol 180: 6117-6125.

Kaper, J.B., Morris, Jr, J.G., and Levine, M.M. (1995) Cholera. Clin Microbiol Rev 8: 48-86.

Karaolis, D.K.R., Johnson, J.A., Bailey, C.C., Boedeker, E.C., Kaper, J.B., and Reeves, P.R. (1998) A Vibrio cholerae pathogenicity island associated with epidemic and pandemic strains. Proc Natl Acad Sci USA 95: 31343139.

Karaolis, D.K., Somara, S., Maneval, Jr, D.R., Johnson, J.A., and Kaper, J.B. (1999) A bacteriophage encoding a pathogenicity island, a type-IV pilus and a phage receptor in cholera bacteria. Nature 399: 375-379.

Kovach, M.E., Shaffer, M.D., and Peterson, K.M. (1996) A putative integrase gene defines the distal end of a large cluster of ToxR-regulated colonization genes in Vibrio cholerae. Microbiology 142: 2165-2174.

Lin, Z., Kumagai, K., Baba, K., Mekalanos, J.J., and Nishibushi, M. (1993) Vibrio parahaemolyticus has a homolog of the Vibrio cholerae toxRS operon that mediates environmentally induced regulation of the thermostable direct hemolysin gene. J Bacteriol 175: 3844-3855.

Linn, T., and Pierre, R.S. (1990) Improved vector system for constructing transcriptional fusions that ensure independent translation of LacZ. J Bacteriol 172: 1077-1084.

Maeda, S., and Mizuno, T. (1990) Evidence for multiple OmpR-binding sites in the upstream activation sequence of the ompC promoter in Escherichia coli: a single OmpRbinding site is capable of activating the promoter. $J$ Bacteriol 172: 501-503.

Martínez-Hackert, E., and Stock, A.M. (1997) Structural relationships in the OmpR family of winged-helix transcription factors. J Mol Biol 269: 301-312.

Mekalanos, J.J., Swartz, D.J., Pearson, G.D., Harford, N., Groyne, F., and de Wilde, M. (1983) Cholera toxin genes: nucleotide sequence, deletion analysis and vaccine development. Nature 306: 551-557.

Miller, J.H. (1972) Experiments in Molecular Genetics. Cold Spring Harbor, NY: Cold Spring Harbor Laboratory Press.

Miller, V.L., and Mekalanos, J.J. (1984) Synthesis of cholera toxin is positively regulated at the transcriptional level by ToxR. Proc Natl Acad Sci USA 81: 3471-3475.

Miller, V.L., and Mekalanos, J.J. (1988) A novel suicide vector and its use in construction of insertion mutations: 
osmoregulation of outer membrane proteins and virulence determinants in Vibrio cholerae requires ToxR. J Bacteriol 170: 2575-2583.

Miller, V.L., Taylor, R.K., and Mekalanos, J.J. (1987) Cholera toxin transcriptional activator ToxR is a transmembrane DNA binding protein. Cell 48: 271-279.

Neely, M.N., Dell, C.L., and Olson, E.R. (1994) Roles of LysP and $\mathrm{CadC}$ in mediating the lysine requirement for acid induction of the Escherichia coli cad operon. J Bacteriol 176: 3278-3285.

Ottemann, K.M., DiRita, V.J., and Mekalanos, J.J. (1992) ToxR proteins with substitutions in residues conserved with $\mathrm{OmpR}$ fail to activate transcription from the cholera toxin promoter. J Bacteriol 174: 6807-6814.

Peterson, K.M., and Mekalanos, J.J. (1988) Characterization of the Vibrio cholerae ToxR regulon: identification of novel genes involved in intestinal colonization. Infect Immun 56: 2822-2829.

Pfau, J.D., and Taylor, R.K. (1996) Genetic footprint of the ToxR binding site in the promoter for cholera toxin. Mol Microbiol 20: 213-222.

Pfau, J.D., and Taylor, R.K. (1998) Mutations in toxR and toxS that separate transcriptional activation from DNA binding at the cholera toxin gene promoter. $J$ Bacteriol 180: 4724-4733.

Pratt, L.A., and Silhavy, T.J. (1996) Identification of base pairs important for OmpR-DNA interaction. Mol Microbiol 17: $565-573$.

Reich, K.A., and Schoolnik, G.K. (1994) The light organ symbiont Vibrio fischeri possesses a homolog of the Vibrio cholerae transmembrane transcriptional activator ToxR. $J$ Bacteriol 176: 3085-3088.

Ross, W., Gosink, K.K., Salomon, J., Igarashi, K., Zou, C., Ishihama, A., et al. (1993) A third recognition element in bacterial promoters: DNA binding by the $\alpha$ subunit of RNA polymerase. Science 262: 1407-1413.

Sambrook, J., Fritsch, E.F., and Maniatis, T. (1989) Molecular Cloning: a Laboratory Manual. Cold Spring Harbor, NY: Cold Spring Harbor Laboratory Press.

Sheridan, S.D., Benham, C.J., and Hatfield, G.W. (1998) Activation of gene expression by a novel DNA structural transmission mechanism that requires supercoiling-induced DNA duplex destabilization in an upstream activating sequence. J Biol Chem 273: 21298-21308.
Shen, J., and Gunsalus, R.P. (1997) Role of multiple ArcA recognition sites in anaerobic regulation of succinate dehydrogenase (sdhCDAB) gene expression in Escherichia coli. Mol Microbiol 26: 223-236.

Skorupski, K., and Taylor, R.K. (1997) Cyclic AMP and its receptor protein negatively regulate the coordinate expression of cholera toxin and toxin-coregulated pilus in Vibrio cholerae. Proc Natl Acad Sci USA 94: 265-270.

Slauch, J.M., and Silhavy, T.J. (1996) The porin regulon: a paradigm for the two-component regulatory systems. In Regulation of Gene Expression in Escherichia coli. Lin, E.C.C., and Lynch, A.S. (eds). Austin, TX: R. G. Landes, pp. 383-417.

Sperandio, V., Girón, J.A., Silveira, W.D., and Kaper, J.B. (1995) The OmpU outer membrane protein, a potential adherence factor of Vibrio cholerae. Infect Immun 63: 4433-4438.

Sperandio, V., Bailey, C., Girón, J.A., DiRita, V.J., Silveira, W.D., Vettore, A.L., and Kaper, J.B. (1996) Cloning and characterization of the gene encoding the OmpU outer membrane protein of Vibrio cholerae. Infect Immun 64: 5406-5409.

Suck, D. (1994) DNA recognition by DNase I. J Mol Recognition 7: 65-70.

Taylor, R.K., Miller, V.L., Furlong, D.B., and Mekalanos, J.J. (1987) Use of phoA gene fusions to identify a pilus colonization factor coordinately regulated with cholera toxin. Proc Natl Acad Sci USA 84: 2833-2837.

Tow, L.A., and Coyne, V.E. (1999) Cloning and characterisation of a novel ompB operon from Vibrio cholerae 569B. Biochim Biophys Acta 1444: 269-275.

Waldor, M.K., and Mekalanos, J.J. (1996) Lysogenic conversion by a filamentous phage encoding cholera toxin. Science 272: 1910-1914.

Welch, T.J., and Bartlett, D.H. (1998) Identification of a regulatory protein required for pressure-responsive gene expression in the deep-sea bacterium Photobacterium species strain SS9. Mol Microbiol 27: 997-985.

Yang, Y., and Isberg, R.R. (1997) Transcriptional regulation of the Yersinia pseudotuberculosis $\mathrm{pH} 6$ antigen adhesion by two envelop-associated components. Mol Microbiol 24: 499-510. 